\title{
Dietary quality of adults in a large metropolitan area of the Kurdistan Region, Iraq
}

\author{
J.S. Ismail, L. O’Connor and S. Patel \\ Department of Health Professions, Manchester Metropolitan University, Manchester, M15 6BG
}

Populations undergoing rapid economic development often adopt adverse lifestyle behaviours including changes to dietary intakes that then affect subsequent chronic disease risk ${ }^{(1)}$. Assessment of dietary intakes and quality are of paramount importance in such populations, to determine population risk for developing nutrient deficiency and chronic diseases and subsequent development of effective intervention strategies. There is a paucity of previous research available on dietary intakes and quality of the adult population living in Erbil city of Kurdistan Region of Iraq (KRI). The aim of this cross-sectional study was to assess the dietary intakes and diet quality in this rapidly developing geographical region.

A multidimensional questionnaire was used to collect data on demographic characteristics, including age, sex and socioeconomic status (SES) [composite measure including house ownership, dwelling type, number of occupants, income and education level).Food consumption was assessed using a modified NHANES 2009-2010 dietary screener. Fruit and vegetable, wholegrain foods and pulse intakes were considered as high diet quality foods and sugar-sweetened beverages, processed meats and energy-dense food consumption were considered as low diet quality foods ${ }^{(2,3)}$. The data collected were characterised using descriptive statistics. Independent $t$ test and one-way ANOVA were used to ascertain differences in intakes of high and low diet quality foods by demographic characteristics.

Of 300 questionnaire distributed, 122 were completed (41\% response rate) and included for analyses. $55.7 \%(n=68)$ of participants were male, the majority (36.7\%) of the sample were aged 18-28 years and $44.6 \%$ listed their highest completed education level as college/institute. Total high quality food consumption was [mean (standard deviation)] $6.8(1.8)$ servings per day, total low quality food consumption was $6.0(2.8)$ servings per day. Age, sex and SES influenced diet quality, see table below.

Table Variation of high and low diet quality score with demographic characteristics $(n=122)$

\begin{tabular}{|c|c|c|c|c|c|c|c|}
\hline \multirow[b]{3}{*}{ Demographic characteristic } & & \multicolumn{6}{|c|}{ Intakes, servings per day } \\
\hline & & \multicolumn{3}{|c|}{ High diet quality foods } & \multicolumn{3}{|c|}{ Low diet quality foods } \\
\hline & & Mean & SD & $\mathrm{P}$ value & $\overline{\text { Mean }}$ & $\mathrm{SD}$ & $\mathrm{P}$ value \\
\hline Age & $18-28(n=44)$ & $6 \cdot 7$ & $2 \cdot 1$ & $>0.05$ & $6 \cdot 4$ & $3 \cdot 1$ & $>0.05$ \\
\hline \multirow{2}{*}{ (years) } & $29-39(\mathrm{n}=37)$ & $7 \cdot 0$ & $1 \cdot 5$ & & $6 \cdot 1$ & $2 \cdot 1$ & \\
\hline & $\geq 40(\mathrm{n}=39)$ & $6 \cdot 8$ & $1 \cdot 7$ & & $5 \cdot 6$ & $3 \cdot 1$ & \\
\hline \multirow[t]{2}{*}{ Sex } & Men $(n=68)$ & $6 \cdot 8$ & 1.8 & $>0.05$ & $6 \cdot 7$ & $2 \cdot 8$ & 0.004 \\
\hline & Women $(n=54)$ & $6 \cdot 9$ & $2 \cdot 1$ & & $5 \cdot 2$ & $2 \cdot 5$ & \\
\hline \multirow[t]{3}{*}{ SES } & Lowest $(n=37)$ & $7 \cdot 2$ & $2 \cdot 0$ & $>0.05$ & $6 \cdot 7$ & $2 \cdot 9$ & $>0.05$ \\
\hline & Middle $(n=44)$ & $6 \cdot 7$ & $1 \cdot 6$ & & $6 \cdot 1$ & $2 \cdot 8$ & \\
\hline & Highest $(n=41)$ & $6 \cdot 6$ & $1 \cdot 7$ & & $5 \cdot 2$ & $2 \cdot 4$ & \\
\hline
\end{tabular}

SD: standard deviation; SES: socioeconomic status

Considering the individual foods within these composite diet quality scores, the oldest age group consumed more whole grains and less processed meat compared to the other age groups $(p=0.001$ and $p=0.01$ respectively). Women consumed fewer servings per day of sugar sweetened beverages compared to men $(p=0.001)$ and the highest SES group consumed fewer daily servings of processed meat $(\mathrm{p}=0.03)$.

This study is the first of its kind to undertake nutritional research in the rapidly changing area of Erbil, KRI. The results demonstrate that age, sex and SES inequalities in terms of dietary quality exist in Eribil. In order to improve the diet quality of this population and reduce the risk of chronic disease, public health campaigns aimed at improving dietary quality and reducing socio-demographic inequalities are warranted.

1. Popkin BM, Adair LS, Ng SW (2012) Nutr Rev 70, 3-21.

2. Wirt A, Collins CE (2009) Public Health Nutr 12, 2473-92.

3. Waijers PM, Feskens EJ, Ocke MC (2007) Br J Nutr 97, 219-31. 